\title{
Éric ALARY, La Grande Guerre des civils
}

Paris, Perrin, coll. Tempus, 2018, 472 pages

\section{Michel Cadé}

\section{(2) OpenEdition}

\section{Journals}

Édition électronique

URL : https://journals.openedition.org/questionsdecommunication/21637

DOI : 10.4000/questionsdecommunication.21637

ISSN : 2259-8901

Éditeur

Presses universitaires de Lorraine

\section{Édition imprimée}

Date de publication : 31 décembre 2019

Pagination : 338-341

ISBN : 9782814305632

ISSN : 1633-5961

Référence électronique

Michel Cadé, "Éric ALARY, La Grande Guerre des civils », Questions de communication [En ligne], 36 | 2019, mis en ligne le 31 décembre 2019, consulté le 30 juin 2022. URL : http://journals.openedition.org/ questionsdecommunication/21637; DOI : https://doi.org/10.4000/questionsdecommunication. 21637 
la marchandise devient l'horizon unique de l'art et du luxe » (p. 102). Après ces très intéressantes premières remarques, le reste du chapitre est étrangement consacré à des analyses qui semblent plutôt concerner le domaine artistique que les liens entre l'art et le luxe, laissant le lecteur quelque peu sur sa faim...

In fine, ce petit livre s'avère - et c'est bien dommage déconnecté de toute démarche et investigation ethnologiques, pourtant promises par le titre. Quelque peu décevant pour le lecteur possédant déjà une connaissance minimale sur le domaine du luxe, il peut néanmoins constituer une première approche commode et accessible à tous ceux intéressés par les questions liées au luxe, mais qui ne connaîtraient pas les analyses et les données existant sur ces questions. Toutefois, on peut tirer profit de la thèse en sciences de l'information et de la communication de Véronique Anderlini-Pillet, intitulée L'Artification du luxe dans le discours médiatique: des signes au sens (2010-20 I4), à l'université Nice Sophia Antipolis et soutenue en 2016.

Clara Lévy

Cresppa-LabToP, université Paris 8-Vincennes-Saint-Denis F-93526 clara.lewy05[at]univ-paris8.fr

\section{Éric Alary, La Grande Guerre des civils \\ Paris, Perrin, coll. Tempus, 2018, 472 pages}

Version « poche », légèrement remaniée, d'un ouvrage paru chez le même éditeur en 20 3, La Grande Guerre des civils sinscrit dans le mouvement de commémoration de la guerre de 1914-1918 et les recherches historiques qu'il a suscitées. Ouvrage de synthèse, il interroge un pan d'histoire paradoxalement insuffisamment étudié, le monde des civils, l'arrière (du front) mais aussi les territoires occupés. En effet, les témoignages et les études sur l'univers des tranchées comme celui des offensives dévoreuses d'hommes sont extrêmement nombreuses (p. 9), la vie de ceux qui restaient en dehors des champs de bataille, du front, a suscité beaucoup moins d'intérêt dans la mesure où elle fut corrélée au moral des troupes, le fameux « pourvu quil|ls tiennent », et, face à l'horreur et la misère vécues par les poilus, considérée comme un moindre mal. Pour faire simple, elle ne manquait peut-être pas de grandeur mais à tout le moins de panache et constitua un sujet médiocre pour les romanciers et pour les historiens, il y eut des exceptions (p. 13), entrainés malgré eux? - dans les délices du roman national. Éric Alary construit son travail dans une double démarche personnelle - il s'est intéressé au quotidien des Français entre 1939 et 1949 dans un précédent ouvrage publié en 2009 (p. 12), et collective -, le renouveau de l'histoire de la « vie quotidienne » (p. I3) et celui des recherches sur la Grande Guerre, avec ses polémiques et ses nouveaux chantiers (pp. 14-16). L'ouvrage est organisé de façon thématique, avec en début et fin, des chapitres plus inscrits dans la chronologie.

Les trois premiers chapitres ( $"$ La fin des jours ordinaires » [pp. 21-63]; « Hommes et femmes à chacun son front » [pp. 65-86]; « Des lendemains douloureux » [pp. 87-129]) dressent une sorte d'état des lieux. D'abord, la surprise d'une guerre à laquelle on ne s'attend pas, surtout dans les campagnes qui se vident de leurs hommes (p. 37) tandis qu'on laisse parfois la moisson en plan (pp. 34-35). Si la guerre n'était, en général, pas prévisible, la haine de l'Allemand - qui surprend Stéfan Zweig (pp. 23-24) - explique un enthousiasme en général partagé, dont des personnes aux noms à consonance allemande ou des entreprises suisses comme Maggi font parfois les frais (pp. 29-33). Rien d'étonnant à ce que les trains de mobilisés partent dans un grand concours de «À Berlin !», ponctués de chants patriotiques (pp. 40-42). Mais le cœurn'y est pas toujours (p. 40). La légitimité du conflit n'est quasiment pas remise en cause (p. 45) et l'« Union sacrée » est la règle, au début d'une guerre que l'on croit rapide et gagnée d'avance (pp. 5 I-52). En ce début de guerre, les enfants appréhendent celle-ci sur un mode à la fois ludique et angoissé (pp. 52-57) et nombre d'entre eux vont devoir prendre part aux travaux des champs quand l'école se fait école du patriotisme sans nuances (pp.59-63). Les hommes partis, les bras manquent pour faire tourner l'industrie, comme l'agriculture (les 2/5e des paysans sont au front) (p. 67). Tout naturellement, les femmes jouent dans l'économie un rôle primordial, que ce soit à la campagne - comme le souligne en 1924, Ernest Pérochon dans Les Gardiennes (pp. 70-7I), roman porté à l'écran par Xavier Beauvois en 2017, centenaire oblige-dans le commerce (p. 75) ou à l'usine (p. 77). Si l'emploi des femmes comme ouvrières n'est pas une nouveauté, son extension à tous les secteurs, particulièrement à la métallurgie (p. 80), modifie une division du travail genrée. Les femmes vont prendre une part considérable aux soins des blessés transportés à l'arrière (pp. 81-82). Et se retrouvant provisoirement chefs de famille, elles vont être paradoxalement des pères de substitution (pp. 83-86), tandis que le gouvernement, en créant la franchise postale, donne, dans l'échange continu des lettres, une réalité virtuelle aux familles (pp. 85-86). La réalité de la guerre moderne, avec son cortège de morts innombrables, s'impose malgré la censure et le bourrage de crânes. La conviction d'une guerre courte est remisée au rang des idées reçues distillées par une propagande au définitive inefficace, car démentie par les faits : la victorieuse bataille de la Marne et la course à la mer ont fait en 
l'espace de 5 mois près de 300000 tués dans les rangs français : 2000 morts par jour (p. 87), soit par mois plus de morts que lors de la bataille de Verdun, repère habituel de la brutalité du conflit. Rappeler l'hécatombe des premiers mois de la guerre, certes bien connue des historiens, ainsi que le poids des 600000 prisonniers faits par les Allemands en 1914-1915, est sans doute pour le grand public une nécessité pour comprendre la mise en place d'une société de guerre où les sacrifices vont s'accroissant, quand certains services, particulièrement de santé, vont diminuant (p. 91). Par les hôpitaux mis en place loin du front pour soigner les blessés, la guerre sinvite dans les villes et les campagnes: le pays est parcouru de trains de blessés, des femmes volontaires participent aux soins (pp. 96-102). D'une certaine façon, l'arrière et le front coïncident. Sans doute, cela rend-il d'autant plus douloureux, pour les familles endeuillées, le transferre du front vers les usines travaillant pour la défense nationale d'ouvriers qualifiés nécessaires à l'effort de guerre, les affectés spéciaux (pp. 94-95). Du fait des bombardements de la région parisienne et Calais par voie aériennne ou par de très grosses pièces d'artillerie, la guerre s'invite d'une autre façon à l'arrière. Commencés en 1914, les raids aériens s'accentueront tout au long de la guerre. Cette violence à l'égard des civils atteint son acmé lors du début de la guerre, en Belgique et dans la zone des combats dans la Nord et l'Est de la France, où les armées allemandes ont multiplié les exactions, liées selon leurs chefs au souvenir des francs-tireurs de 1870. D'une guerre l'autre... (pp. I 19-127). Devenues un thème de propagande, ces « atrocités allemandes 》, dont la destruction de Reims est la plus emblématique (p. I06-I |9) sont cependant bien réelles, comme le confirme l'historienne Annette Becker (p. 124). Elles marqueront durablement les mémoires et leur souvenir ne sera pas pour rien dans l'exode de juin 1940 (p. I29).

Les chapitres 4 et 5 (« Exodes et évacuations 》 [pp. | |31 - I54] et « L'heure allemande » [pp. 155- 187]) constituent sans doute l'apport le plus original de cet ouvrage destiné à un large public, car ils sintéressent à des pans d'histoire en général évacués des ouvrages traitant de la Grande Guerre : l'exode d'habitants des zones occupées ou situées sur le front et la vie des Français sous administration allemande. Pour nombre de lecteurs, ils seront une découverte. Historien de la France de la Seconde Guerre mondiale, Éric Alary est sensible aux similitudes, souvent peu soulignées, entre les deux conflits. Ainsi sintéresse-t-il à la masse considérable des réfugiés partis à la dérive sur les routes de France en août 1914: 2 millions de personnes, majoritairement belges, mais tout de même 640000 Franç̧ais (p. I33) qui ne retrouveront leur domicile, souvent détruit, qu'après l'armistice de novembre 1918. Non anticipé, ce mouvement s'est déroulé dans le plus grand désordre (pp. 135-137). La panique gagne même Paris avant la victoire de la Marne le 6-9 septembre 1914 (pp. | 140-145). Les réfugiés se jugent souvent abandonnés (p. 153), voire pour les Alsaciens Lorrains mal accueillis (p. 15 I). D'autant que, les Allemands expulsant une partie des habitants des régions qu'lls occupent, le flot ne se tarit pas (p. I52). Cependant, la solidarité nationale semble dans bien des cas avoir joué de façon positive (p. 154). À la fin de l'année 19|4, 8 départements sont occupés totalement ou partiellement (p. 155). Les habitants y vivent privés de liberté et dans des conditions économiques difficiles, souvent victimes d'une répression sévère (emprisonnements, exécutions, listes d'otages à fusiller en cas d'opposition marquée à l'occupation allemande, et d'exactions diverses [pp. 163 170]). Il est un peu dommage que l'auteur ait inclus dans les territoires occupés ceux d'Alsace-Lorraine, cédés aux Allemands en 187| (pp. |69-17I). Ici, pas dinvasion mais une région de l'Empire allemand depuis plus d'une génération, 43 ans en 1914 ! Si le sentiment pro-français y est assurément important, cela ne justifie pas cette vision téléologique du passé qui se confirme dans le titre de la dernière partie du chapitre 5 « Répétition générale: "s'accommoder", collaborer, résister» (pp. | 86- | 87).Tout au long du chapitre, des termes anachroniques comme 《z zone libre » (p. 179) ou « Français libres » (p. 186) témoignent de cette dérive.

Levée l'hypothèque des territoires occupés, les cinq chapitres suivants (« Privations» [pp. 189-227], « Le rose et le noir» [pp. 279-250], « Fragiles retrouvailles et infidélités 》 [pp. 251-290], «Une société recomposée » [pp. 191-316], « Oublier la guerre » [pp. 317-335]) sont consacrés à ce qu'on eût appelé dans les années 1960 « la vie quotidienne des Français de l'arrière pendant la Grande Guerre ». Médiocrité des rations alimentaires avec de nombreuses disparités territoriales (pp. 201-205), hausse des prix - qui eût gagné à être exprimée en francs constants dans le tableau de la page 200 -, raréfaction de produits jugés de première nécessité (pp. 206-208), contrôle étatique face au système $D$ (pp. 215-217) et profiteurs en tout genre (pp. 223-227) tracent un tableau convainquant des effets de la guerre sur les consommateurs.

Plus original, le chapitre 7 interroge le poids de la mort sur la société française et les moyens de ne pas s'abandonner au prévisible, mais encore non advenu, malheur. La mise en évidence d'un immense lien de courrier et de colis jamais sans doute n'a-t-on tant écrit en France (p. 230) -, ourre des perspectives historiographiques nouvelles. C'est ce flux constant, entre le front et l'arrière, qui maintient debout les familles et d'une certaine façon la nation, tout en conjurant l'omniprésence de la mort (pp. I30- 
237).Vienne celle-ci, le deuil impose une retenue où la souffrance individuelle se transcende, du moins dans ses manifestations publiques, dans un deuil collectif (pp. 237238). Pourtant, chacun craint la venue du gendarme ou du maire porteur de mauvaise nouvelle, mission délicate que certains assurent avec empathie (pp. 240-242). Tandis que se multiplient les veuves mal soutenues par l'État (pp. 242 243), les enfants paient le prix fort, celui d'une psyché traumatisée (p. 245).L'emprise sociale des morts est telle, qu'en 1916, Maurice Barrès propose qu'ils exercent leur droit de vote par l'entremise de leur veuve, proposition discutée en 1922-1923 (pp. 248-249) ; en 1919, Abel Gance leur avait donné la parole dans son film J'accuse (pp. 247-248). Les morts, les pauvres morts, pèseront lourd sur la France de l'entre-deux-guerres.

Évoquer de façon croisée les fragiles retrouvailles et l'infidélité est un des acquis de l'histoire présente. Longtemps, le second terme fut tabou. Qu'on se souvienne de l'accueil ambigu et indigné qui fut réservé à l'ouvrage de Raymond Radiguet Le Diable au corps en 1923 comme en 1947 au film éponyme de Claude Autant Lara. Les retrouvailles sont moins merveilleuses que rêvées (p. 252). Linncommunicable expérience du front, le désir sexuel exacerbé (p. 255), les changements vestimentaires (p. 270), les inévitables soupçons après de long mois de séparation (p. 272), le désordre des sens (p. 275), les amis trop prévenants (p. 276), tout concourt à ces cas limites qui se terminent par un « crime passionnel » (p. 275). Même s"lls ne paraissent pas avoir été la règle, des cas attestés comme celui de Roland Dorgelès et de Mado, largement cité dans l'ouvrage (pp. 276-280), montrent qu'lls furent sans doute plus communs qu'on ne crut. II faudrait mesurer l'effet des conditions sociales, comme du contrôle social, pour sortir d'un certain flou. Outre que les choses semblent se passer mieux dans les cas de familles construites, outre aussi que les parents et particulièrement la mère, sont, autant que l'épouse et les enfants, des éléments clé des retrouvailles (pp. 252-265), les « marraines de guerre » redonnent aux jeunes femmes une aura dont les soupçons individuels avaient pu les priver collectivement (pp. 285-290).

Les changements quinnduit la guerre dans la société sont d'ordre divers. La démographie se trouve bouleversée : surmortalité chez les hommes jeunes; baisse des mariages puis redressement à partir de 1918 (p. 292) ; augmentation des divorces à partir aussi de 1918 (pp. 293-296) - plus conséquence du traumatisme des combattants que de l'adultère féminin (p. 296) - ; chute de la natalité qui peine en 1919 à retrouver les chiffres de 1914 (p. 298) ; enfin, déséquilibre des sexes (en 1921, I 100 femmes pour I 000 hommes, p. 30I). La mobilité sociale est sur l'échelle des fortunes totale. Souvent légitimes, les profits de guerre voient les « nouveaux riches $»$, industriels comme intermédiaires, tenir le haut du pavé, dont les plus marquants sont André Citroën, Louis Loucheur, les Berliet (p. 304) quand la baisse des salaires des employés, les pertes de nombre de rentiers ou demirentiers transforment une partie de la petite et moyenne bourgeoisie en nouveaux pauvres (p. 308). Le fait est patent, d'autant que le départ sous les drapeaux du chef de famille a souvent abouti à un déclassement (p. 310 ). En termes d'économie comme de consommation, la guerre a plutôt profité au monde rural (pp. 3/2-3/4) mais n'a pas enrayé l'exode rural (p. 3/4). Le monde ouvrier enregistre un très léger mieux (p. 315) cependant il doit accepter la taylorisation dont André Citroën se fait le chantre (p. 3|6). Parfois peu lisibles pendant le conflit, ces modifications vont entrainer dans les années suivantes de véritables changements structurels.

Entrée dans le quotidien, la guerre ne pouvait demeurer le seul horizon. Un réveil religieux fut le premier refuge, et la foi le moyen de transcender l'épreuve (pp. 318-322). Pourtant, dès 1915 , on retrouva le goût de se distraire. Le sport semble avoir connu une éclipse (p. 324). La mode transforma, avec la fin du corset, la vie comme l'apparence des femmes (p. 325). Et surtout, on retrouva le chemin des lieux de distraction (théâtres, music-hall, cabaret, cinéma) et pas seulement à Paris, ville sur laquelle l'auteur fonde essentiellement ses analyses (pp. 326-328). Malgré des conditions d'exploitations difficiles, théâtre et music-hall sont pleins à Paris comme à Bordeaux, Nantes ou Perpignan (pp. 327-330). Le moyen d'évasion le plus populaire, car le moins onéreux et le plus varié, est le cinéma. Jamais les cinémas ne furent plus fréquentés, d'autant que, contrairement à ce qu'affirme Éric Alary, les bandes projetées n'étaient pas toutes, loin de là, d'une piètre qualité et que la projection avait rapidement retrouvé un bon niveau (p. 330). S'agissant toujours du cinéma, l'auteur, sans trop s'égarer, manque un peu de références. Les Mystères de New York (p. 332) est bien un serial américain mais produit par une firme française, Pathé, son rival n'est pas Les Mystères de Paris mais un film Gaumont, sorti un peu avant en novembre 1915, Les Vampires de Louis Feuillade, qui devait récidiver en janvier 1917 avec Judex, immense succès. Le jugement de valeur sur le cinéma des années de guerre - « le plus souvent médiocre » (p. 332) - gagnerait à être nuancé, tout comme la phrase sur Abel Gance à être précisée. Les premiers films italiens sont à l'affiche avant la guerre. Enfin, le « septième art » n'est pas né pendant la Grande Guerre (p. 332) mais, tout en sachant que la première projection date de 1895 , il accède au statut d'art dès 1908 avec le « film d'art » même si aujourd'hui l'on peut considérer que le cinéma de Méliès était un cinéma 
d'art. Ce genre d'inexactitudes n'est pas anormal dans un ouvrage de synthèse, il est moins acceptable dans une réédition. Quant aux enfants, ils lisent Bécassine [qui est née en 1905, et non en 1915, et est une domestique, non une fermière, bretonne (p. 333)] et jouent... à la guerre (pp. 334-335).

Les deux derniers chapitres (« De guerre lasse » [pp. 337-378] et « Le jour d'après [pp. 379-403]) traitent des derniers moments du conflit. Abordant les années 1917 et 1918, l'auteur entremêle avec intelligence événements militaires et vie civile. Un hiver 1916-1917 extrêmement froid rend dans tout le pays le quotidien des Français douloureux (p. 344), surtout les efforts excessifs demandés aux ouvriers (p. 339) ; et la saignée de l'offensive du Chemin des Dames au printemps 1917 (p.340) entraînent une véritable dépression nationale qui ne se traduit pas des grèves (p.347-350) et des mutineries (p. 340). Si, à l'occasion, le gouvernement est mis sur la sellette par certains organes de presse (pp. 34I-343), le contrôle de l'opinion s'accentue via le contrôle postal et la censure (pp. 350-353). L'arrivée des Américains - qui ne va pas sans troubles (pp. 253-256) -, comme celle de Georges Clemenceau à la tête du gouvernement en novembre 1917 (p. 337), contribuent à redresser la situation malgré lintensification des bombardements sur la région parisienne (pp. 358-362). Les reculs alliés du printemps 1918 qui entrainent un nouvel exode (pp.362366) et un mouvement de grève parallèle font douter, sans ébranler, un consensus national retrouvé. La victoire efface, un instant, le souvenir de ces temps difficiles dans l'euphorie de grandes fêtes spontanées dans tout le pays (pp. 367-375), même si, très vite, va se poser le problème des régions libérées en partie dévastées (pp. 374-378). Le début de l'après-guerre commence mal avec l'épidémie mortifère de grippe espagnole (pp. 38I-384) qui ajoute de nouveaux morts à ceux du conflit ( I 295 000) (p. 386). La démobilisation, lente, n'est pas un simple acte technique car encore faut-il réintégrer ceux qui vont devenir les « anciens combattants 》, ce qui, hormis pour les paysans, est souvent complexe malgré les nécessités de la reconstruction (pp. 386-392). Mutilés et blessés comme les prisonniers rencontrent des difficultés spécifiques et l'amertume est grande chez ceux qui doivent réapprendre à vivre, diminués pour bon nombre d'entre eux (pp. 392-396). Redevenir civil est aussi un apprentissage qui se fait parfois au détriment des femmes - qui quittent leurs emplois de temps de guerre (pp. 296-297) - et de l'équilibre des familles (pp. 397-398). Quant aux régions libérées, leur retour à la normale est sans cesse repoussé (pp. 399-103). Pour les civils nouveaux et anciens, les effets de la guerre se prolongent jusqu'au début des années 1920. Mais, de façon plus pernicieuse, ils ne s'achèveront que dans le conflit suivant, dont l'auteur - il n'est pas seul -, postule la continuité ( $p .410)$, sans cependant valider la thèse d'une guerre de 30 ans (p.412).

Synthèse indispensable, en général bien au fait des travaux historiques les plus récents, avec un a priori accordé à la thèse du consentement - l'école de Péronne pour faire court -, face à celle de la contrainte - le Collectif de recherche international et de débat sur la guerre de 1914-1918 - qui eut mérité d'être explicité, fondé sur une connaissance des sources solide, La Grande Guerre des civils est un ouvrage utile tant pour les historiens, les enseignants du secondaire, les étudiants et, de façon plus générale, pour tous ceux qui cherchent un guide leur facilitant la compréhension des œuvres de fiction qui évoquent la Grande Guerre vue de l'arrière romans, bandes dessinées, films. L'appareil critique qui l'accompagne et une solide bibliographie permettront à celles et ceux qui veulent pousser plus loin la réflexion de le faire avec efficacité. En somme, un ouvrage de vulgarisation nécessaire et un peu plus...

$$
\begin{array}{r}
\text { Michel Cadé } \\
\text { Cresem, université de Perpignan Via Domitia, F-66860 } \\
\text { cade[at]univ-perp.fr }
\end{array}
$$

\section{Éric AnCEau, Henri Temple, dirs, Qu'est-ce qu'une nation en Europe?}

Paris, Sorbonne université presses, coll. Sorbonne essais, 2018, 332 pages

L'ouvrage réunitcontributions de spécialistes européens de l'étude de la nation, qui concerne la période qui va de 1789 jusqu'à Emmanuel Macron. Si la tonalité de cet ensemble est bien française, l'approche quant à elle, est bel et bien européenne : Robert Tombs, historien de la France et de la Grande-Bretagne, fournit un texte bien utile à un ensemble où les nombreux auteurs français comportent des spécialistes de pays tels la Roumanie et l'Espagne. Spécialiste de la Russie, Françoise Thom apporte une vision de l'extrême-est européenne de la question. Les aspects juridiques sont traités par HenriTemple et Olivier Gohin ; «la dimension spatiotemporelle de la nation » (pp. 45-95) par le géographe Jean-Pierre Doumenge. Pour sa part, l'économiste Jean-Pierre Werrebrouck traite de l'euro, « ce talisman qui a détruit les nations » (pp. 165-182), tandis que Pierre-André Taguieff présente «l'Europe souveraine » d'Emmanuel Macron (pp. 283-324). En bref,à la suite de l'introduction des deux directeurs de l'ouvrage, douze contributions traitent des multiples dimensions de la nation et l'Europe. 